\title{
Lage und Zukunft der Kriminologie - Fragen und Antworten
}

\author{
Prof. Dr. Hans-Jörg Albrecht, Max-Planck-Institut für ausländisches und internationales \\ Strafrecht, Freiburg
}

\section{Wie bewerten Sie die aktuelle Lage und Bedeutung der Kriminologie in Deutschland?}

Die aktuelle Lage der Kriminologie ist problematisch. Dabei ist nur wenig zu differenzieren. Während die Kriminologie an den Universitäten in den letzten 20 Jahren erheblich an Bedeutung verloren hat, kann für die Kriminologie an Fachhochschulen (insbesondere der Polizei) und an Landeskriminalämtern ein Bedeutungszuwachs festgestellt werden. Damit ist allerdings eine im Hinblick auf Forschung und Beruf spezialisierte Kriminologie verbunden. An den Rechtswissenschaftlichen Fakultäten wird eine Kriminologie, die empirisch ausgerichtet ist und strafrechtswissenschaftliche, soziologische, psychologische, ökonomische und psychiatrische Perspektiven einbezieht, dagegen eher randständig. Kriminologie wird heute ganz überwiegend (in der Auszeichnung von Lehrstühlen) mit den klassischen Fächern des Strafrechts kombiniert und bildet sich in einen juristisch geprägten Annex zurück. Dies ist verständlich angesichts eher geringer Stellenzahlen im Strafrecht und den Lehr- und Prüfungsaufgaben in den Kernfächern. Der Anspruch einer aus einer interdisziplinären Bewegung entstandenen Kriminologie, Fortschritt in der permanenten Integration von Wissen und der Einbeziehung von Disziplinen, die aus je eigenen Perspektiven relevante Fragestellungen aufgreifen, zu suchen, lässt sich so nicht mehr erfüllen. Aus den soziologischen Instituten und Fakultäten ist die Kriminologie (oder Kriminalsoziologie) fast vollständig verschwunden. Dasselbe gilt für psychologische Institute, geht es um die Rechts- und Kriminalpsychologie. Die Forensische Psychiatrie befasst sich zwar wieder stärker mit kriminologischen Fragestellungen (vor allem in der Entwicklung von aktuarischen Prognoseinstrumenten und in der Untersuchung von Gefährlichkeit sowie Behandlung), ist aber ansonsten an der Kriminologie wenig interessiert. Dies hat auch zur Folge, dass sich empirische kriminologische Forschung heute auf wenige Institute konzentriert, im Übrigen handelt es sich dabei weitgehend um die Institute, die bereits in den 1970er Jahren die deutsche Kriminologie vertreten haben. Dass die Kriminologie „als Schnittmenge von Kriminalsoziologie, -psychologie, -psychiatrie u. ä.“ heute regelmäßig durch einen Lehrstuhl in der juristischen Fakultät vertreten werde, wie Bernd Schünemann dies in einer Antwort auf den Wissenschaftsrat zu den Zukunftsperspektiven der (Straf)Rechtswissenschaften angenommen hat, ${ }^{1}$ erscheint deshalb selbst bei einer äußerst gutwilligen Betrachtung der Lage als schlicht nicht (mehr) zutreffend.

1 Schünemann, Standpunkte der deutschen Strafrechtslehrer zu den Zukunftsperspektiven der Rechtswissenschaft und der akademischen juristischen Ausbildung in Deutschland. Zeitschrift für Internationale Strafrechtsdogmatik 2012, S. 302-311. 
Wie würden Sie die Bedeutung der deutschen Kriminologie im internationalen Vergleich einordnen?

Die Bedeutung der deutschen Kriminologie ist im internationalen Vergleich gestiegen und hat - nicht in Ausbildungsangelegenheiten, aber in der theoretischen und empirischen Forschung - ein hohes Niveau. Die deutsche Kriminologie ist heute deutlich sichtbarer in der internationalen (und englischsprachigen) Literatur sowie auf europäischen und internationalen Konferenzen als noch in den 1960er und 1970er Jahren. Die Beiträge der deutschen Kriminologie zur Längsschnitt- und Lebenslaufforschung, zur Evaluation und Implementierung von Kriminalpolitik, zu transnationaler Kriminalität und internationaler Verbrechenskontrolle sowie zum internationalen Vergleich belegen eine an internationalen Standards orientierte methodische und theoretische Stärke und ferner gerade im Vergleich zur anglo-amerikanischen Kriminologie - betrachtet man dortige neuere Selbstvergewisserungsversuche ${ }^{2}$ - einen interdisziplinären Vorsprung, der nicht zuletzt aus einer engeren (und effektiveren) Kooperation mit dem Strafrecht und einer sehr viel intensiveren strafrechtswissenschaftlichen Grundlagenforschung resultiert. Hinzu kommt, dass sich die deutschen kriminologischen Zeitschriften internationalisieren, sichtbar auch an der Entwicklung der Monatsschrift für Kriminologie und Strafrechtsreform. Die deutsche Kriminologie ist ferner europäisch und international gut vernetzt und in vergleichenden und auf Kooperation angelegten Untersuchungen ebenso gut vertreten.

\section{Wo sehen Sie die Stärken und Schwächen der kriminologischen Ausbildung in Deutschland?}

Die kriminologische Ausbildung (im Jurastudium) beschränkt sich auf höchstens 2 (zweistündige) Vorlesungen, die in der Regel in einen Schwerpunktbereich fallen, der auch Strafvollzugsrecht, Jugendstrafrecht und strafrechtliche Sanktionen umfasst. Dies reicht nicht einmal im Ansatz aus für eine Ausbildung in Methoden und Statistik, die eigentlich neben der Ausbildung in Theorie und Theorieentwicklung einen Ausbildungsschwerpunkt darstellen müssten. Kriminologische Ausbildung reduziert sich damit auf Veranstaltungen mit Einführungscharakter. Da allerdings Masterstudiengänge für Kriminologie in Deutschland nur unzureichend angeboten werden (Hamburg, Bochum, Greifswald, mit je eigenen Schwerpunktsetzungen) kann von einer Stärke kriminologischer Ausbildung in Deutschland eigentlich nicht gesprochen werden. Wenn eine Stärke (im internationalen Vergleich) vorliegt, dann ist es die weiter oben bereits angesprochene Nähe zum Strafrecht und die effektive Aktivierung des hier angelegten Wissens. Eine starke kriminologische Ausbildung bedürfte allerdings differenzierter Post Graduate Qualifizierungsangebote, die den Anspruch der Kriminologie, empirische und interdisziplinär angelegte Wissenschaft zu sein, sowohl in Curricula wie auch im Lehrpersonal effektiv implementieren. Ansätze hierzu waren in der einspurigen Juristenaus-

2 Bosworth/Hoyle (Hrsg.), What is Criminology?, 2011.

NK 25. Jg. $1 / 2013$ 
bildung der 1970er Jahre, bspw. mit der Ansiedlung von Soziologen in Juristischen Fakultäten, vorhanden, sind aber Vergangenheit.

\section{Wo seben Sie auch in der Zukunft bedeutsame und gesellschaftlich sowie politisch relevante kriminologische Forschungsfelder?}

In den in der Zukunft bedeutsamen Forschungsfeldern hat die Kriminologie erhebliche, wenn auch nicht unbedingt überlegene Konkurrenz bekommen. Sicherheit und Gefährlichkeit, extreme und kollektive Gewalt, Schattenwirtschaften, Terrorismus, zerbrochene Staaten, Wiederaufbau von staatlichen Strukturen und von Monopolen der Gewalt, internationale Kriminalität sowie formalisierte und informelle transnationale Systeme der Kontrolle beziehen sich eigentlich auf zentrale Fragestellungen der Kriminologie. Jedoch sind es weitgehend andere Disziplinen, die sich wie Politik- und Sozialwissenschaften, Internationale Beziehungen, Ethnologie - und dies weitgehend ohne besondere Rückbindung an den kriminologischen Bestand an theoretischem und empirischem Wissen - nunmehr solchen Fragen widmen.

Ferner zeigt sich, dass Ökonomie und Psychologie (teils kombiniert mit der Gehirnforschung) in den letzten Jahren verstärkt Fragen aufgreifen, die ebenfalls Grundlagenfragen der Kriminologie berühren. Sie können mit Verweisen auf die experimentelle Forschungen der Behavioral Economy, die Untersuchung von Vergeltung, Rache, Strafe, Bindungen oder Selbstkontrolle nur kurz benannt werden. Aus diesen Ansätzen erwächst auch ein erhebliches Potenzial für eine interdisziplinäre Kriminologie, die so zu den Akteuren und zu den Grundlagen von Handlungen, werden sie nun als Täter- oder Opferhandlungen eingestuft, neue Ansätze entwickeln kann.

Die Kriminologie hat sich lange Zeit und in erheblichem Umfang mit Sachverhalten abgegeben, deren Relevanz sich aus dem Massencharakter verschiedener Formen der Alltagskriminalität ergab. Dabei sind vor allem Sachverhalte der Schattenwirtschaften und der Unterwelt in den Hintergrund getreten. Die informelle Ökonomie der Drogen, des Rotlichts, gestohlener oder unverzollter Waren oder scharf kontrollierter Dienstleistungen ist verbunden mit der Entwicklung der Großstädte und von in prekären Verhältnissen lebenden sozialen Gruppen , ${ }^{3}$ andererseits Ausdruck von Globalisierung und Internationalisierung auch der informellen Ökonomie . ${ }^{4}$ Dort sind Einsichtsmöglichkeiten in Phänomene angelegt, die mit Begriffen wie organisierte Kriminalität, Verbrechen als Beruf und kriminelle Netzwerke nur unzureichend erfasst werden und die von der deutschen Kriminologie bislang kaum aufgegriffen werden.

Schließlich noch ein Hinweis auf die empirische Strafverfahrens- und Strafvollstreckungsforschung, die wenig ausgebildet ist. ${ }^{5}$ Der signifikante Wandel, der sich partiell

3 Shapland/Ditton/Godefroy/H. J. Albrecht (Hrsg.), The Informal Economy: Threat and Opportunity in the City, 2003.

4 Paoli/Greenfield/Reuter, The World Heroin Market - Can Supply Be Cut?, 2009.

5 Albrecht, Rechtstatsachenforschung zum Strafverfahren. Empirische Untersuchungen zu Fragestellungen des Strafverfahrens zwischen 1990 und 2003, 2005. 
deckt mit der Hinwendung zu einem Sicherheitsstrafrecht, wird weitgehend aus einer strafrechtlichen und normativen Perspektive untersucht. Dies gilt für die Veränderungen in den Ermittlungspraktiken ebenso wie für die neuen Arrangements zwischen Strafrecht, Strafvollstreckung und Polizeirecht, die die Figur des "Gefährders" geschaffen haben.

\section{Prof. Dr. Jochen Bung, Universität Passau}

Wie bewerten Sie die aktuelle Lage und Bedeutung der Kriminologie in Deutschland?

Mit Blick auf die Praxis ergibt sich ein ambivalentes Bild. Es zeigt sich eine gewisse Verengung des institutionell relevanten kriminologischen Forschungsinteresses auf Sexualund Gewaltstraftaten, was aber natürlich auch in der institutionellen „Logik“ der Verwahranstalten begründet ist. An den Hochschulen, namentlich den juristischen Fachbereichen, ist das Interesse der Studierenden am kriminologischen Schwerpunktstudium (inkl. Jugendstrafrecht, Strafvollzug und Sanktionenlehre) unverändert hoch. Wo Kriminologie angeboten wird - leider längst nicht mehr überall - sind stabil hohe Besucherzahlen zu verzeichnen.

Wie würden Sie die Bedeutung der deutschen Kriminologie im internationalen Vergleich einordnen?

Die lebendigsten Diskussionen finden schon seit einer Weile in der US-amerikanischen Kriminologie statt. Dort wird die deutsche Kriminologie aber kaum zur Kenntnis genommen, was deren Bedeutung nicht ganz gerecht wird.

Wo sehen Sie Stärken und Schwächen der kriminologischen Ausbildung in Deutschland?

Kriminologie ist eine vielschichtige und verzweigte Disziplin mit vielen Verbindungen $\mathrm{zu}$ anderen Wissenschaften, vielleicht besteht sie sogar geradezu aus diesen Verbindungen. Als Appendix-Fach im Rahmen des juristischen Schwerpunktstudiums - und nur darüber kann ich einigermaßen verlässlich Auskunft geben - kann sie allerdings kaum mehr als ein paar Schlaglichter auf einige interessante Fragen werfen. Inhaltlich sehe ich die ausbildungsbedingte Nähe zum Strafrecht eher als Vorteil, wenn man aufpasst, dass kritische, kriminalpolitische und kriminalsoziologische Perspektiven nicht zu kurz kommen. 
Wo sehen Sie auch in der Zukunft bedeutsame und gesellschaftlich sowie politisch relevante kriminologische Forschungsfelder in Bezug auf...

a) ...die Untersuchung der Täter („Verbrecher“)?

Das fachspezifische aber auch medienöffentliche Interesse an Themen wie Sicherungsverwahrung, Jugendkriminalität und Hirnforschung zeigt, dass klassisch kriminalätiologischen Fragestellungen dauerhafte Konjunktur beschieden sein wird.

b) ...die Untersuchung von Deliktsphänomenen („Verbrechen“)?

Da die Phänomenologie dieser Phänomene nicht aus diesen selbst entwickelt werden kann, subsumiere ich dieses Forschungsfeld unter c).

c) ...die Untersuchung der staatlichen und gesellschaftlichen Reaktion auf Kriminalität („Verbrechenskontrolle“)?

Hier ist im Allgemeinen ein Rückgang des Interesses zu verzeichnen, was womöglich auf ein Paradox zurückzuführen ist: Je mehr die Gesellschaft sich zur Kontrollgesellschaft entwickelt, desto geringer erscheint das Reflexionsbedürfnis in Bezug auf diese Entwicklungen. Schade, denn hier ist das eigentliche, womöglich wichtigste kriminologische Forschungsfeld.

d) ...die Untersuchung der Rolle der Verbrechensopfer?

Mit dem Bedeutungsverlust gesellschaftsbezogener Fragestellungen hängt die Karriere des Opfers als Gegenstand kriminologischen Interesses zusammen. Das wird, flankiert nicht zuletzt durch die regierende Kriminalpolitik, noch eine Weile so weitergehen. Innerkriminologisch handelt es sich um das logische Komplement zur Täterkriminologie. Eine wichtige Ergänzung, solange man den Gesamtzusammenhang nicht aus dem Blick verliert.

\section{Prof. Dr. Heinz Cornel, Alice Salomon Hochschule, Berlin}

\section{Wie bewerten Sie die aktuelle Lage und Bedeutung der Kriminologie in Deutschland?}

Der Aufbruch der Kriminologie mit der Gründung mehrerer kriminalsoziologischer Lehrstühle in den siebziger Jahren, verbunden mit der Hoffnung, die Kriminologie werde sich als eigenständige, wenn auch interdisziplinär verbundene Wissenschaft in Deutschland etablieren können, ist nicht nur beendet. Die Abwicklung zahlreicher kriminologischer Lehrstühle hat zugleich zu einer noch festeren Anbindung an die juristischen Fachbereiche geführt. Das stellt für die großen kriminologischen Lehrstühle insoweit 
kein Problem dar, als sie in interdisziplinären Teams sozialwissenschaftliche Kompetenz einbeziehen können. Viele andere können das nicht. Die Masterstudiengänge der Kriminologie bieten neue Chancen interdisziplinärer kriminologischer Ausbildung auf hohem Niveau.

Wie würden Sie die Bedeutung der deutschen Kriminologie im internationalen Vergleich einordnen?

Ich kann und möchte da ungern Zensuren verteilen. Die eingeschränkte Eigenständigkeit der Kriminologie ist sicherlich im Verhältnis zur britischen und US-amerikanischen ein Standortnachteil. Gleichwohl würde ich aus der Tatsache, dass deutsche Kriminologen eher englischsprachige kriminologische Publikationen zur Kenntnis nehmen als umgekehrt, nicht auf eine geringere Bedeutung deutscher Kriminologie schließen. Dass ein Teil der deutschen kriminalpolitischen und insbesondere strafrechtlichen Praxis kriminologische Begleitforschung nicht für unverzichtbar hält, ist für den langjährigen Beobachter auffällig, aber wohl im internationalen Vergleich kein Alleinstellungsmerkmal.

\section{Wo sehen Sie die Stärken und Schwächen der kriminologischen Ausbildung in Deutschland?}

Die oben schon genannte Tatsache, dass es keine eigene grundständige Ausbildung der Kriminologie in dafür eingerichteten Fachbereichen für Forschung und Lehre in Deutschland gibt, ist nicht nur eine Schwäche an sich, sondern muss auch zu einer differenzierten Beantwortung der Frage führen. Dabei überblicke ich bei weitem nicht alle einschlägigen Studiengänge, denn kriminologische Ausbildung wird sicherlich auch an medizinischen und psychologischen Fachbereichen beispielsweise geleistet. Es braucht wohl auch keine besonderen internen Einblicke, um feststellen zu können, dass nicht alle Strafrechtslehrstühle an allen juristischen Fachbereichen Deutschlands die Ausbildung der Kriminologie in ihrer Interdisziplinarität und Aktualität auf gleich hohem Niveau leisten können. Für die Mehrheit der Studiengänge der Sozialen Arbeit und Sozialpädagogik an Fachhochschulen und Universitäten sind keine Kriminologen berufen, obwohl Abweichendes Verhalten und Delinquenz sowie die Reaktion darauf in den Curricula eine große Rolle spielen. Der seit knapp drei Jahren aktive ,Arbeitskreis der HochschullehrerInnen für Kriminologie/Straffälligenhilfe in der Sozialen Arbeit' hat etwa 30 hauptamtlich lehrende Personen mit zahlreichen Lehrformaten erfasst - in vielen der Bachelorstudiengänge findet eine kriminologische Ausbildung aber kaum statt. In den sozialpädagogischen Bachelorstudiengängen der Universitäten sieht es ähnlich aus, jedoch mag da für die Studierenden fachbereichsübergreifend eine kriminologische Ausbildung leichter zugänglich sein. Meines Wissens wird davon häufig kein Gebrauch gemacht. Auf die positiven Aspekte der Masterstudiengänge der Kriminologie sei auch diesbezüglich nochmals hingewiesen. 
Wo sehen Sie auch in der Zukunft bedeutsame und gesellschaftlich sowie politisch relevante kriminologische Forschungsfelder in Bezug auf...

a) ...die Untersuchung der Täter („Verbrecher“)?

Es würde der deutschen Kriminologie gut tun, wenn sozialstrukturelle, konstruktivistische, labelingtheoretische und individualpsychologische Erklärungsansätze in ihrer Relevanz für die Untersuchung der Täter bzw. der Zuschreibungsprozesse hinsichtlich der Täter zusammen gesehen würden (nicht im Sinne gar zu schlichter Mehrfaktorenansätze) und entsprechend auch interdisziplinär gegebenenfalls unter Einbeziehung von Medizin, Pädagogik und Ökonomie gearbeitet würde. Hochinteressant fände ich eine Neudiskussion, Überprüfung und Aktualisierung der frühen - vor allem US-amerikanischen kriminalsoziologischen Klassiker von Merton über Cohen/Short bis Sykes/Matza.

b) ...die Untersuchung von Deliktsphänomenen („Verbrechen“)?

Neben der ewigen Relevanz der Gewalt- und Drogendelinquenz, die sich allein schon aus den gesellschaftlichen Reaktionen ergibt, halte ich die Entwicklung einer Kriminologie der Gefährdungsdelikte für bedeutsam. Die kriminologische Forschung hält da meines Erachtens mit dem strafrechtlichen und rechtsoziologischen Diskurs nicht mit. Hinweisen möchte ich auch auf die Makrokriminalität, die Kriminalität der Mächtigen, die meines Erachtens nur allzu sporadisch thematisiert wird.

c) die Untersuchung der staatlichen und gesellschaftlichen Reaktionen auf Kriminalität (,Verbrechenskontrolle“)?

Aus meiner Perspektive möchte ich hier insbesondere Jugendhilfe im Jugendstrafverfahren, Untersuchungshaft, Strafvollzug, Sicherungsverwahrung, Bewährungshilfe, Führungsaufsicht und freie Straffälligenhilfe nennen, staatliche Reaktionen und Institutionen, die nicht nur im Spannungsfeld von Repression, Kontrolle und Hilfe stehen, sondern kriminalpolitisch auch Aufgaben und Funktionen jenseits kriminologischer Wirksamkeitsanalysen zugewiesen bekommen. Das kann man einerseits selbst untersuchen hinsichtlich der Hypothesen über das Verhältnis von Sozial- und Kriminalpolitik oder von neuer Punitivität und der Abkehr vom Wohlfahrtsstaat, wie das in unterschiedlichsten Varianten von Franz von Liszt bis David Garland geschah. Andererseits können die einzelnen staatlichen und gesellschaftlichen Reaktionen und die sie tragenden oder hervorbringenden Institutionen selbst aber auch im Einzelnen hinsichtlich ihrer Wirkungen im Prozess der Kriminalisierung und gegebenenfalls Resozialisierung und Integration kriminologisch untersucht werden. 


\section{Prof. DrJörg Kinzig, Universität Tübingen}

\section{Wie bewerten Sie die aktuelle Lage und Bedeutung der Kriminologie in Deutschland?}

Wenn ich es richtig sehe, sind die aktuelle Lage und die Bedeutung der Kriminologie in Deutschland von gegenläufigen Tendenzen geprägt. Während die institutionelle Situation des Faches Kriminologie, insbesondere an den Universitäten, auf der einen Seite als prekär bezeichnet werden kann, hat die Bedeutung kriminologischer Themen im gesellschaftlichen Diskurs auf der anderen Seite in den letzten Jahren erheblich zugenommen.

a) Die institutionelle Situation des Faches Kriminologie: Wie bekannt, ist in Deutschland, ob berechtigt oder nicht, das Fach Kriminologie primär an den Juristischen Fakultäten lokalisiert und wird dort dem Strafrecht zugeschlagen. Schon „das Strafrecht“ leidet bei den Juristen (der Einfachheit halber benutze ich in dieser Stellungnahme männliche Formen, wohlwissend, dass die Nachfrage in den kriminologischen Schwerpunktbereichen ganz überwiegend von Studentinnen ausgeht) aber als neben dem Zivil- und Öffentlichen Recht kleinstes Fach häufig in besonderer Weise, wenn es - was nicht selten ist - an Juristischen Fakultäten zu Stellenkürzungen kommt. Innerhalb des Strafrechts gerät dann vielfach die Kriminologie unter besonderen Legitimationsdruck und muss im Ergebnis hinter scheinbar wichtigeren, moderner anmutenden und auch lukrativeren Ausrichtungen wie z.B. dem Wirtschaftsstrafrecht oder dem Europäischen und Internationalen Strafrecht zurücktreten.

Die universitäre Lage in den anderen Bezugsdisziplinen der Kriminologie wie etwa der Soziologie, der Psychologie und der Psychiatrie kann ich nicht vollständig beurteilen. Sie scheint mir aber nach allem, was ich auf der insoweit verdienstvollen Tagung des Freiburger Max-Planck-Instituts im Sommer 2012 zu der Situation der Kriminologie in Deutschland gehört habe, eher noch problematischer zu sein.

Vor diesem Hintergrund empfinde ich es als anachronistisch, dass sich die deutsche Kriminologie national immer noch die Organisation in zwei, wenn auch unterschiedlich bedeutsame Wissenschaftsvereinigungen leistet. Gerät das Fach Kriminologie als solches unter Druck, wäre das Gebot der Stunde, alle vorhandenen Kräfte zu bündeln.

b) Die gesellschaftliche Bedeutung des Faches Kriminologie: Diesem insgesamt zu konstatierenden institutionellen Bedeutungsverlust steht eine ungeheure, nach meinem Empfinden eher noch wachsende Nachfrage nach kriminologischer Expertise in der Gesellschaft gegenüber. Besonders deutlich wird dieser Befund, wenn man sich anschaut, wie vielen kriminologischen Fragestellungen sich vor allem die Medien tagtäglich zuwenden. So gehen bei mir - und vielen Kollegen wird es ähnlich ergehen - zahlreiche mediale Anfragen aller Art ein - von Lokalredakteuren der Heimatzeitung bis hin zu denen von Fernsehjournalisten - die demonstrieren, dass die Kriminologie offensichtlich ein gesellschaftlich höchst relevantes Themenfeld beackert.

Darüber, warum man es hier mit der paradoxen Situation einer steigenden gesellschaftlichen Nachfrage bei gleichzeitig sinkendem institutionellem Angebot zu tun hat, kann man nur spekulieren. Offensichtlich ist es uns Kriminologen bisher nicht gelungen, aus dem wachsenden Bedarf Kapital zu schlagen. $Z u$ vermuten ist auch, dass ein zu hohes 
Maß kriminologischen Sachverstands nicht in den Zeitgeist passt und daher auch von den (politischen) Entscheidungsträgern nicht gewollt ist.

\section{Wie würden Sie die Bedeutung der deutschen Kriminologie im internationalen Vergleich einordnen?}

Die deutsche Kriminologie hat im internationalen Vergleich nicht die Bedeutung, die ihr nach der Größe und Wirtschaftskraft des Landes eigentlich zukommen sollte. Gründe für diesen Umstand gibt es meines Erachtens mehrere:

Bereits oben wurde die in den letzten Jahren schlechtere Verankerung des Faches an den Universitäten, insbesondere an den Juristischen Fakultäten, hervorgehoben. Damit sind unmittelbar Rückwirkungen auf den kriminologischen Nachwuchs verbunden. Wer sich heute an Juristischen Fakultäten habilitieren will, kann nur davor gewarnt werden, sich ausschließlich der Kriminologie zu widmen. Fast überall ist das Standbein, das (dogmatische) Straf- und Strafprozessrecht, für eine Karriere zu einer (dann vielleicht auch kriminologisch ausgerichteten) Universitätsprofessur wichtiger als das Spielbein Kriminologie. Dies führt dazu, dass ein Habilitand schon den Spagat zwischen dem Allgemeinen Teil des StGB und der Kriminologie (unter Einschluss von Jugendstrafrecht und Strafvollzug) beherrschen muss. Kriminologie auch international zu betreiben, ist daneben kaum möglich.

Zudem führt die allein in Deutschland vorhandene große Nachfrage nach kriminologischem Wissen dazu, dass sich schon in unserem Land für einen Kriminologen ein reichhaltiges Betätigungsfeld ergibt. Darüber hinaus ist für einen „Juristen-Kriminologen" das nach wie vor national geprägte Strafrecht der wichtigste Bezugspunkt. Abhilfe ist hier meines Erachtens nur dann zu erwarten, wenn das Fach Kriminologie an universitären und außeruniversitären Einrichtungen deutlich gestärkt wird.

\section{Wo sehen Sie Stärken und Schwächen der kriminologischen Ausbildung in Deutschland?}

Die Verankerung der Kriminologie an den Juristischen Fakultäten hat aus meiner Sicht Vor- und Nachteile. Trotz der Nachteile halte ich kriminologisch ausgerichtete Lehrstühle an den Juristischen Fakultäten für unverzichtbar.

Der Vorteil der Lokalisierung der Kriminologie bei den Juristen liegt zum einen darin, dass künftige Strafrichter, Rechtsanwälte, Ministerial- und höhere Polizeibeamte etc. so zumindest eine Chance haben, sich schon während des juristischen Studiums mit kriminologischen Fragestellungen vertraut zu machen. Zudem muss die Kriminalpolitik wenigstens die Möglichkeit haben, sich von in Strafrecht und Kriminologie versierten Hochschullehrern beraten zu lassen.

Bedauerlich ist es, dass es an den sozialwissenschaftlichen Fakultäten noch weniger Lehrstühle zu geben scheint, die sich mit abweichendem Verhalten in seiner ganzen Breite beschäftigen. Dies ist auch deswegen bedauerlich, weil somit der unerlässliche sozial- 
wissenschaftliche Input in die in Deutschland von Juristen dominierte Kriminologie zu versiegen droht.

Wo sehen Sie auch in der Zukunft bedeutsame und gesellschaftlich sowie politisch relevante kriminologische Forschungsfelder in Bezug auf...

(Die Frage ist angesichts der derzeitigen schnellen gesellschaftlichen Veränderungen nur schwer zu beantworten. Zudem dürfte meine Prognose von meinen eigenen Forschungsinteressen geprägt sein).

a) ...die Untersuchung der Täter („Verbrecher“)?

Als Tätergruppen, die zunehmend in den kriminologischen Fokus rücken könnten, wage ich zu prognostizieren: politisch und religiös motivierte Terroristen, Sexualstraftäter (als derzeitige Inkarnation des „Bösen“), Wirtschaftsstraftäter (bei vermutlich sich weiter verschärfenden Gegensätzen zwischen Arm und Reich), jugendliche Intensiv- sowie (angesichts der demographischen Entwicklung) auch ältere Straftäter.

b) ... die Untersuchung von Deliktsphänomenen („Verbrechen“)?

Die möglicherweise zunehmend kriminologisch interessierenden Deliktsphänomene korrespondieren zum Teil mit den unter a) genannten Tätergruppen: dazu gehören Großverbrechen aller Art (des Staates, aber auch von einzelnen), der Bereich der Internetkriminalität, Sexual- und Wirtschaftsstraftaten, Armutskriminalität sowie spezielle Ausprägungen der Gewaltkriminalität (etwa von Jugendlichen oder im Sport etc.).

c) ... die Untersuchung der staatlichen und gesellschaftlichen Reaktion auf Kriminalität („Verbrechenskontrolle“)?

Ein adäquates Vorgehen gegen gefährliche Straftäter (Stichwort: Sicherungsverwahrung) wird uns voraussichtlich noch eine ganze Weile beschäftigen. In diesem Zusammenhang wird die Maßregel der Führungsaufsicht mit den sie begleitenden Weisungen (Stichwort: „elektronische Fußfessel“) weiter an Bedeutung gewinnen. Der erhebliche Anstieg der psychiatrisch Untergebrachten macht es darüber hinaus erforderlich, dass auch der Anwendung dieser Sanktion vermehrt kriminologische Aufmerksamkeit geschenkt wird.

Auch könnte der Kostendruck dazu führen, dass im Bagatellbereich verstärkt auf ein ambulantes Vorgehen gesetzt wird, dessen Effektivität zu untersuchen wäre.

Abzuwarten bleibt, inwieweit neuartige Sanktionen Einzug in das strafrechtliche Arsenal halten werden, die dann auch auf kriminologisches Interesse stoßen sollten. 
d) ... die Untersuchung der Rolle der Verbrechensopfer?

Wenn ich es richtig sehe, nimmt die Bedeutung der Rolle der Verbrechensopfer im strafrechtlichen Verfahren unverändert zu. Diese Entwicklung mit ihren Rückwirkungen auf den Tatverdächtigen, später unter Umständen dann auch strafrechtlich Angeklagten, muss meines Erachtens von der kriminologischen Forschung kritisch begleitet werden.

\section{Privatdozent Dr. Reinhard Kreissl, Universität Wien}

Wie bewerten Sie die aktuelle Lage und Bedeutung der Kriminologie in Österreich?

Österreich ist ein kleines Land, hat weniger Einwohner als Bayern, aber doch immerhin 9 Bundesländer. Wien ist das Zentrum des Landes (Sicht der Wiener), bzw. der Wasserkopf der Republik (so sehen es die Anderen). Im Hinblick auf die intellektuelle und akademische Landschaft konzentriert sich sehr viel in der Hauptstadt. Es gibt an der juristischen Fakultät der Universität Wien mittlerweile einen als Kriminologen ausgewiesenen Kollegen, dessen Licht aber bisher nicht sehr weit leuchtete. Nennenswerte bzw. wahrnehmbare empirische kriminologische Forschung wird an der Universität in Linz betrieben. Rechtssoziologisch bemerkenswerte Beiträge kommen immer wieder von der Universität Innsbruck. Darüber hinaus ist wenig zu vermelden. Der Status der Kriminologie an den Universitäten ist marginal, es bieten sich keine Karrierechancen und daher ist das Fach für die helleren Köpfe unter den Studierenden wenig attraktiv. Das derzeit von mir geleitete Institut für Rechts- und Kriminalsoziologie war unter der Leitung des verstorbenen Heinz Steinert in seiner inzwischen 40-jährigen Geschichte eine Zeit lang über die Grenzen des Landes hinaus sichtbar, beschränkt sich heute aber im Hinblick auf kriminologisch relevante Forschung im Wesentlichen auf Arbeiten für das österreichische Ministerium der Justiz und ist auf dem akademischen Publikationsmarkt kaum präsent.

Kriminologische Agenden scheinen auch hierzulande in den Bereich der Fachhochschulen ausgewandert zu sein. In den sozialpädagogisch ausgerichteten Fachbereichen werden insbesondere im Hinblick auf Fragen der Jugendkriminalität immer wieder kleinere Projekte initiiert. An den sozialwissenschaftlichen Fakultäten der Universitäten taucht Kriminologie praktisch nicht auf.

Wie würden Sie die Bedeutung der österreichischen Kriminologie im internationalen Vergleich einordnen?

... zu vernachlässigen. 
Wo sehen Sie Stärken und Schwächen der kriminologischen Ausbildung in Österreich?

Es gibt keinen eigenständigen Ausbildungsgang (MA oder Dipl.) für Kriminologie, und dementsprechend tut sich auf dem Sektor wenig. Der typische Kriminologe ist hierzulande - wie in anderen kontinentalen Ländern - der Jurist mit minimalen statistischen Kenntnissen und bestenfalls rudimentär informiert über die Standardtheorien, die sich in den Lehrbüchern der Kriminologie finden. Da weder eine Professionalisierung der Kriminologie stattgefunden hat noch eine akademisch-intellektuelle Forschungstradition existiert, gibt es auch keine spezifische Ausbildung, die Schwächen oder Stärken ausbilden könnte. Kriminologie ist ein Orchideennebenfach der juristischen Fakultäten.

Wo seben Sie auch in der Zukunft bedeutsame und gesellschaftlich sowie politisch relevante kriminologische Forschungsfelder in Bezug auf...

a) ...die Untersuchung der Täter („Verbrecher“)?

Da weiß man inzwischen wohl alles, was wissenswert ist. Wechselnde Moden (wie derzeit der neuro- oder biowissenschaftliche turn) führen zu Reformulierungen alter Einsichten, bringen aber keinen nennenswerten Erkenntnisgewinn.

b) ...die Untersuchung von Deliktsphänomenen („Verbrechen“)?

Hier beschränkt sich die Forschung auf das Nachakademisieren tagesaktueller Populismen - von Cyber-Crime bis Kinderpornografie. Interessant wäre eine eher kulturtheoretische Interpretation des Wechsels in der gesellschaftlichen Aufmerksamkeit für bestimmte Phänomene, die als „Verbrechen“ Konjunktur haben. Dafür aber ist die Kriminologie kognitiv nicht ausgerüstet.

c) ...die Untersuchung der staatlichen und gesellschaftlichen Reaktion auf Kriminalität („Verbrechenskontrolle“)?

Würde die Kriminologie bei Durkheim über die Normalität des Verbrechens nachlesen, könnte sie ihre Lehrbücher zuklappen und sich mit wichtigeren Dingen beschäftigen. Der Paradigmenwechsel sozialer Kontrolle, die Konjunktur eines Sicherheitsdenkens, das Aufkommen neuer Regulierungsformen, der selektive Einsatz von Strafen, um nur einige Stichpunkte zu nennen, wären für sich genommen interessante Themen, die aber auf den Bildschirmen der Kriminologie nicht auftauchen.

d) ... die Untersuchung der Rolle der Verbrechensopfer?

Das Opfer hat wohl die erstaunlichste Karriere als Thema der Kriminologie in den letzten zwanzig Jahren hingelegt. Warum das so ist und welche gesellschaftlichen Mentalitäten

NK 25. Jg. 1/2013 
das befeuern, ist eine interessante Frage, die aber - s.o. - von der Kriminologie theoretisch nicht begriffen wird. Stattdessen tut sich mit der Viktimologie ein weiteres lukratives Forschungsfeld auf, das Publikationschancen und Fördermittel verspricht.

Insgesamt ist - aber das ist die marginale Meinung eines eher an gesellschaftstheoretischen Fragen interessierten Sozialwissenschaftlers - die Kriminologie auch im 21. Jahrhundert nicht über ihren Geburtsfehler hinweg gekommen, dass sie das Verbrechen am, im oder um den Täter herum sucht. Sie wird es nicht finden und daher gilt: further research is needed!

\section{Prof. Dr. Karl-Ludwig Kunz, Universität Bern}

\section{Wie bewerten Sie die aktuelle Lage und Bedeutung der Kriminologie in der Schweiz?}

Es gibt ein spezielles Studium an der Universität Lausanne, ein Nachdiplomstudium, eine Doktoratsschule und das Veranstaltungen anbietende Berner Forum für Kriminalwissenschaften an der Universität Bern, vielfach an mehreren Universitäten in der juristischen Masterausbildung und als Nebenfach für Nichtjurist(inn)en besuchte universitäre Lehrveranstaltungen, Fortbildungskurse für Strafrechtspraktiker in Luzern, einen viel besuchten jährlichen Kongress der Schweizerischen Arbeitsgruppe für Kriminologie, die mehrmals jährlich erscheinende Schweiz. Zeitschrift für Kriminologie, die manchmal kriminologische Beiträge bietende Schweiz. Zeitschrift für Strafrecht und die zumeist Dissertationen veröffentlichenden Schweiz. Kriminologischen Untersuchungen. Das Fach ist hauptsächlich als Spezialfach bei der Juristenausbildung angebunden. Die großen Universitäten verfügen nur teilweise über einen strafrechtlichen Lehrstuhl, der auch Kriminologie unterrichtet. Die akademische Forschung zeigt sich in allen Sparten von Veröffentlichungen, wobei ein gewisser Schwerpunkt bei praxisnahen Dokumentationen erkennbar ist. Eigene kriminologische Lehrstühle gibt es nicht. In den Kontexten von Soziologie und Psychologie spielt die Kriminologie in Lehre und Forschung eine eher untergeordnete Rolle. Die Forschung ist zumeist regional begrenzt und praxisnah. Während das Fach früher akademisch im Rahmen einer verbreitet konsentierten „Gesamten Strafrechtswissenschaft" und praktisch im Rahmen der Gesamtreform des Allgemeinen Teils des Schweiz. Strafgesetzbuches Beachtung erheischte, schwinden inzwischen beide Antriebskräfte. Insbesondere ist das Interesse der Gesetzgebung an kriminologischer Information erlahmt. Mit eher geringer gesellschaftlicher Beachtung suchen Fachvertreter im Spannungsfeld zwischen anwendungsbezogener und theoretischer Forschung nach einer Standortbestimmung. Eine einheitliche Richtung ist bis jetzt nicht erkennbar.

\section{Wie würden Sie die Bedeutung der schweizerischen Kriminologie im internationalen Vergleich einordnen?}

Fachvertreter sind auf der Höhe der Zeit und wirken an internationalen Projekten zahlreich mit. Als früher angeblich „kriminalitätsarmes“ Land zieht die Schweiz vor allem 
aus den USA Aufmerksamkeit auf sich. Die Bedeutung des Fachs ist durch ein Nationales Forschungsprogramm über Gewalt im Alltag und Organisierte Kriminalität gewachsen. Wirtschaftsdelikte und Ausländerkriminalität bilden ein international beachtetes Studienfeld. Als Forschungsfeld entwickelt sich der Bereich zwischen der Kriminalität der Angepassten und moralisch, aber noch nicht rechtlich diskriminiertem Verhalten in Bereichen der Wirtschaft, der Banken und der internationalen in der Schweiz ansässigen Verbände.

Wo sehen Sie Stärken und Schwächen der kriminologischen Ausbildung in der Schweiz?

In gewissem Gegensatz zur gut vollzogenen institutionellen Entwicklung befindet sich der eher schwache methodische Kenntnisstand. Untersuchungen referieren oft naiv über das angeblich vorgegebene Phänomen der Kriminalität und bedienen sich gängiger Analyseinstrumente. Methodische Innovation ist ausgesprochen selten. Forschende können sich mangels Ressourcen und interessierten Personen wenig auf dieses Fach spezialisieren, zumal die Kompetenzen in der Kriminologie neben den vor allem gefragten umfänglichen strafrechtlichen Kompetenzen beschränkt bleiben. Zugleich Nach- wie Vorteile bildet die akademische Anbindung an das juristische Studium. Ausbruchsversuche daraus, etwa das eigene Studium in Lausanne und das Nachdiplomstudium in Bern, sind auch international bemerkenswert. In praxisnahen Fortbildungen und Kongressveranstaltungen wird das Fach problematisch mit der Kriminalistik verbunden.

Wo sehen Sie auch in der Zukunft bedeutsame und gesellschaftlich sowie politisch relevante kriminologische Forschungsfelder in Bezug auf...

a) ...die Untersuchung der Täter („Verbrecher“)?

Karriereforschung, Sanktionswirkungsforschung, Rückfallforschung, Intensivtäter

b) ...die Untersuchung von Deliktsphänomenen („Verbrechen“)?

Ausländerkriminalität, Gewaltstraftaten, Jugendkriminalität, Wirtschaftsdelikte, Kriminalität in internationalen Verbänden

c) ...die Untersuchung der staatlichen und gesellschaftlichen Reaktion auf Kriminalität („Verbrechenskontrolle“)?

Ich stelle dies in den Vordergrund, wobei ich nicht an „einfache“ quantitative Analysen von Statistik und Dunkelfeld denke, sondern vermehrt an anspruchsvolle qualitative und auch theoretische Forschung im Verbund mit weiteren Disziplinen, z. B. Geschichte und Sozialanthropologie. 
d) ...die Untersuchung der Rolle der Verbrechensopfer?

Durch ein spezielles Verwaltungsgesetz (OHG) wurde die Opferhilfe mit Forschungsbedarf ausgebaut. Die Schnittstelle zum Strafrecht verdient vermehrt wissenschaftliche Beachtung.

\section{Prof. Dr. Frank Neubacher, Universität Köln}

Wie bewerten Sie die aktuelle Lage und Bedeutung der Kriminologie in Deutschland?

Die Kriminologie ist eine vom Aussterben bedrohte Disziplin. Ihr Bestand wird meines Erachtens aber weniger durch „die Politik“ oder „die Gesellschaft“ gefährdet, sondern durch Juristische Fakultäten bzw. durch Universitätsleitungen, die den Spardruck abfedern wollen, indem sie sich von kriminologischen Lehrstühlen verabschieden. An zahlreichen Fakultäten gibt es entweder keinen Kriminologen bzw. keine Kriminologin mehr oder die Kriminologie wird von einem Strafrechtler(in) mitverwaltet. Das ist umso weniger zu begreifen, als die Kriminologie sicher zu den von den Studierenden am stärksten nachgefragten Schwerpunktbereichen zählt. Bei Fragen, die die Gesellschaft besonders bedrängen (z.B. zum Strafvollzugsgeschehen, zur Kriminalitätsentwicklung im Hellund Dunkelfeld, zur Erklärung abweichenden Verhaltens, zur Jugendkriminalität) wird nur ein Kriminologe bzw. eine Kriminologin verlässlich Auskunft geben können und mit seiner/ihrer Expertise auch einmal vor Fernsehkameras treten oder ein Zeitungsinterview geben. Wird Wissenschaft ihrer gesellschaftlichen Verantwortung noch gerecht, wenn sie auf gesellschaftlich besonders relevante Fragen die Antwort schuldig bleibt? Sicher nicht! Unbestritten ist auch der Beitrag der Kriminologie zu rechtstatsächlichen Forschungen. Ohne Rückkopplung an empirische Befunde werden die Rechtswissenschaftlichen Fakultäten ärmer sein - zum Schaden der angehenden Juristen und Juristinnen, die sich dann erst in der beruflichen Praxis damit konfrontiert sehen, dass Gesetz und Gesetzesumsetzung häufig zwei ganz verschiedene Dinge sind. Und nicht zuletzt ist (was vor allem aus Sicht der Universitätsleitungen wichtig ist) die Kriminologie vergleichsweise drittmittelstark. Das stärkste Argument für die Kriminologie ist aber die Ausbildungsaufgabe der Fakultäten. Denn wer sonst soll den Strafjuristen erklären, dass Abschreckung nicht einfach deshalb funktioniert, weil sich Juristen das so vorstellen? Wer sonst soll den Blick der Strafjuristen dafür schulen, dass es oftmals nicht die vom Gericht gewählte Sanktion ist, die dem weiteren Lebensweg des Verurteilten eine entscheidende Wendung gibt, sondern Veränderungen in seinem privaten Umfeld? Kurzum: Die schwache Position der Kriminologie wird weder ihrer gesellschaftlichen Bedeutung noch ihrem Stellenwert für die wissenschaftliche Ausbildung an den Juristischen Fakultäten gerecht. Aus der Krise kann meines Erachtens nur die Wiederherstellung des Status quo ante, eine konsequente Nachwuchsförderung und die Schaffung unbefristeter Stellen im Mittelbau helfen, insbesondere für Sozialwissenschaftler, die für die empirischen Forschungsarbeiten unverzichtbar sind. 
Wie würden Sie die Bedeutung der deutschen Kriminologie im internationalen Vergleich einordnen?

Ton und Themen gibt im internationalen Vergleich die angloamerikanische Kriminologie an. Deren Führungsrolle ist unbestritten, führt aber mitunter dazu, dass die Kriminologie in anderen Ländern theoretische und thematische Wendungen mitvollzieht, selbst wenn es sich um zeitgeistige oder kriminalpolitische Verirrungen handelt. Die Stärke der Kriminologie in den USA und in England liegt nicht nur im Sprachenvorteil begründet, aber eine in der Landessprache publizierende europäische Kriminologie wäre niemals im Stande, der amerikanischen Kriminologie ihre Führungsrolle streitig zu machen. Der sehr stark empirisch ausgerichteten angloamerikanischen Kriminologie hat sich in den vergangenen Jahren die niederländische Kriminologie angenähert, die dadurch teilweise an deren führender Position partizipiert. Man wird wohl nicht zu viel wagen, wenn man meint, dass die deutsche Kriminologie (noch?) mitführend ist in Europa. Sie muss meines Erachtens nicht einmal unbedingt zu einer reinen empirischen Wissenschaft werden; auch theoretische Anstöße haben einen hohen Stellenwert. Es hat aber den Anschein, als ob die deutsche Kriminologie selbst insoweit an Strahlkraft eingebüßt hat. In Deutschland droht die Kriminologie in den Status einer „Hilfswissenschaft“ zu degradieren einer Hilfswissenschaft für Strafrechtler, die sich ein wenig empirische Verstärkung wünschen, bzw. für Kriminalpolitiker, die anlassbezogen einmal etwas wissenschaftlich begleitet sehen möchten. Diese Gefahr der Degradierung ist, das sei betont, nicht auf eine theoretische oder empirische Unterentwicklung der Kriminologie zurückzuführen, sondern reflektiert die Ohnmacht einer Disziplin, deren Vertreter sich traditionell in einer doppelten Minderheitenposition wiederfinden, nämlich als Strafrechtler unter Juristen sowie als Kriminologen unter Strafrechtlern.

\section{Wo sehen Sie Stärken und Schwächen der kriminologischen Ausbildung in Deutschland?}

Die Besonderheit der Kriminologie in Deutschland (wie in Kontinentaleuropa) besteht in der Verortung an den Rechtswissenschaftlichen Fakultäten. Eine ihrer Stärken ist es daher, dass sie das kriminologische Wissen dorthin bringt, wo es relevant ist, nämlich zu den angehenden Richtern, Staatsanwälten und Anstaltsleitern. Das geschieht nicht nur in kriminologischen Lehrveranstaltungen, sondern auch in Veranstaltungen zum Jugendstrafrecht und zum Strafvollzugsrecht, also Fächern, die man nur betreiben kann, wenn man rechtsdogmatisch und rechtstatsächlich informiert ist. Eine Schwäche besteht in dem Mangel an empirischer Ausbildung. Hier haben psychologische bzw. soziologische Kriminologen bzw. Kriminologinnen Vorteile gegenüber ihren juristisch ausgebildeten Kollegen. In den USA und in England kann man sehen, dass die Kriminologie nicht notwendigerweise an Juristischen Fakultäten beheimatet sein muss. Gleichwohl spricht sehr viel für die Präsenz der Kriminologie an den Juristischen Fakultäten. Nur so können Recht und Kriminologie in Beziehung gesetzt werden. Ohne diese Verbindung und ohne die rechtswissenschaftliche Expertise gäbe es beispielsweise keine Politikberatung bzw. Mitwirkung bei der Konzeption von Gesetzentwürfen. Hier bewirkt diese Expertise,

NK 25. Jg. $1 / 2013$ 
dass der Kriminologe bzw. die Kriminologin ein ganz anderes Standing in kriminalpolitischen Beratungen hat. Aber zurück zur Ausbildung und ihren Mängeln: Es ist schlicht beschämend, dass in der Strafrechtspraxis zahlreiche Richter und Staatsanwälte nicht auf eine universitäre kriminologische Ausbildung zurückblicken können (eklatant ist z.B. die Situation bei Jugendrichtern und -staatsanwälten, die von den Vorgaben des $\$ 37$ JGG weit entfernt ist). Was soll man also einer Wissenschaft wünschen, deren Kenntnisse zwar sehr stark von Studierenden, der Öffentlichkeit und den Medien sowie Drittmittelgebern nachgefragt werden, die aber vielen Kolleginnen und Kollegen an den Rechtswissenschaftlichen Fakultäten (Köln ausgenommen!) sowie Praktikerinnen und Praktikern gleichermaßen verzichtbar zu sein scheint?

Wo sehen Sie auch in der Zukunft bedeutsame und gesellschaftlich und politisch relevante kriminologische Forschungsfelder in Bezug auf ...

a) ... die Untersuchung der Täter („Verbrecher“)?

Lebenslaufforschung; Auswahl von Tatobjekten bzw. Tatopfern durch Straftäter; Frauenkriminalität

b) ... die Untersuchung von Deliktsphänomenen („Verbrechen“)?

Cybercrime; Organisierte Kriminalität (inkl. globaler Phänomene wie z.B. Menschenhandel); Kriminalitätstheorien (in Deutschland ist die Diskussion hierzu veraltet); sozialer Wandel/Krisenphänomene und Kriminalität

c) ... die Untersuchung der staatlichen und gesellschaftlichen Reaktion auf Kriminalität („Verbrechenskontrolle“)?

Zunehmende „Verpolizeilichung“ (z.B. expansive Rolle der Polizei in „Intensivtäter“Programmen oder Sexualstraftäterdateien), d.h. das Übergreifen der Polizei und des Sicherheitsparadigmas auf Tätigkeitsbereiche, die traditionell Akteuren vorbehalten sind, die rehabilitativ tätig werden (z.B. Jugendhilfe, Schule, Führungsaufsicht); Untersuchung von Einstellungen/Alltagstheorien bei Polizei und Justiz

d) ... die Untersuchung der Rolle der Verbrechensopfer?

Anzeigeverhalten; Wiedergutmachung; Wahrnehmung von Polizei/Justiz 


\section{Prof. Dr. Heribert Ostendorf, Universität Kiel}

Wie bewerten Sie die aktuelle Lage und Bedeutung der Kriminologie in Deutschland?

Das Gewicht der Kriminologie in der strafrechtlichen Ausbildung nimmt ab. Dem entspricht, dass nach meiner Wahrnehmung die Lehrstühle für Kriminologie in Deutschland weniger werden. Umgekehrt hat die Kriminologie in der Politik mit dem Ersten und Zweiten Sicherheitsbericht der Bundesregierung sowie in Antworten der Bundesregierung auf Große Anfragen zur Kriminalpolitik erheblich an Bedeutung gewonnen. Allerdings wird diese allgemeine Orientierung am kriminologischen Sachverstand häufig nicht bei konkreten Gesetzesentscheidungen umgesetzt.

Wie würden Sie die Bedeutung der deutschen Kriminologie im internationalen Vergleich einordnen?

Die Frage ist schwer zu beantworten. Im Vergleich zu den europäischen, insbesondere osteuropäischen Staaten würde ich die deutsche Kriminologie im vorderen Bereich ansiedeln.

Wo sehen Sie die Stärken und Schwächen der kriminologischen Ausbildung in Deutschland?

Die größte Schwäche ist, dass eine kriminologische Ausbildung für Strafjuristen nicht flächendeckend angeboten wird. Auch in den tradierten strafrechtlichen Lehrveranstaltungen müssen zentrale kriminologische Erkenntnisse vermittelt werden. Damit geht einher, dass Lehrveranstaltungen zu den Straftatfolgen, soweit sie überhaupt angeboten werden, für die Studenten keinen Reiz ausüben, da sie mit Ausnahme der Schwerpunktprüfungen kein Prüfungsgegenstand sind.

Wo sehen Sie auch in der Zukunft bedeutsame und gesellschaftlich sowie politisch relevante kriminologische Forschungsfelder?

Den größten kriminologischen Forschungsbedarf sehe ich bei der Frage der staatlichen und gesellschaftlichen Reaktion auf Kriminalität, hier insbesondere in Bezug auf den Wandel des klassischen Strafprozesses zu einem ökonomischen Strafprozess.

\section{Prof. Dr. Tobias Singelnstein, Freie Universität Berlin}

Wie bewerten Sie die aktuelle Lage und Bedeutung der Kriminologie in Deutschland?

Aus meiner Perspektive ist die Lage der Kriminologie prekär. Insbesondere an den juristischen Fakultäten der Universitäten als einer zentralen Stätte kriminologischer For- 
schung und Lehre erlebt das Fach einen erheblichen Bedeutungsverlust und teilt dabei das Los der (anderen) Grundlagenfächer: Im Zuge der überall zu beobachtenden Einsparungen werden kriminologische Lehrstühle ganz gestrichen oder in strafrechtlichkriminologische Lehrstühle umgewandelt. Trotz des ungebrochenen Interesses bei Studierenden leisten sich die Fakultäten eine intensive kriminologische Komponente oft nicht mehr. Dies führt u.a. dazu, dass an den Fakultäten weniger kriminologische, vor allem weniger empirische Forschung betrieben wird. Hierdurch werden insbesondere Grundlagenforschung und solche Forschungsprojekte geschwächt, die von konkreten Verwendungsinteressen unabhängig erfolgen. Zugleich verliert die Kriminologie angesichts dieser eher ungewissen Zukunftsperspektiven auch beim wissenschaftlichen Nachwuchs deutlich an Attraktivität.

Versteht man unter Bedeutung den Einfluss der Kriminologie auf andere Wissenschaften, die öffentliche Debatte und die Politik, ergibt sich ein durchwachsenes Bild. Während die Kriminalpolitik die Ergebnisse kriminologischer Forschung nur selten berücksichtigt, hat die Kriminologie in der öffentlichen Debatte und in anderen Wissenschaften durchaus Spuren hinterlassen, die aber noch deutlich ausgeprägter sein könnten.

Wie würden Sie die Bedeutung der deutschen Kriminologie im internationalen Vergleich einordnen?

Lässt man die dominante Position der anglo-amerikanischen Kriminologie außen vor, so hat die deutsche Kriminologie meines Erachtens keinen schlechten Stand. Dieser wird allerdings durch die beschriebenen Entwicklungen gefährdet, die neben der interdisziplinären auch die internationale Vernetzung und Zusammenarbeit erschweren.

\section{Wo sehen Sie Stärken und Schwächen der kriminologischen Ausbildung in Deutschland?}

Nachdem die Soziologie sozialer Probleme und sozialer Kontrolle an sozialwissenschaftlichen Fakultäten inzwischen kaum noch anzutreffen ist, verliert diese Perspektive in der Kriminologie an Bedeutung. Eine Stärke der kriminologischen Ausbildung besteht demgegenüber in der starken Verankerung an den juristischen Fakultäten in den Schwerpunktbereichen und an den Fachhochschulen. Neben diesen eher knapp gehaltenen Spezialisierungen finden sich auf beiden Seiten Chancen und Räume für andere Formen kriminologischer Ausbildung. So ist zum einen zu überlegen, ob kriminologische Inhalte in einem gewissen Umfang nicht zur Grundlagenausbildung für alle Studierenden der Rechtswissenschaft gehören müssen. Zum anderen sind die Möglichkeiten für eine gezielte, tiefe Spezialisierung in Form von Aufbau- und Masterstudiengängen mit den Angeboten in Hamburg und Bochum bislang nur wenig ausgeprägt. Hierüber ließe sich ggf. auch wieder eine stärkere Verankerung des Faches an sozialwissenschaftlichen Fakultäten erreichen. 
Wo seben Sie auch in der Zukunft bedeutsame und gesellschaftlich sowie politisch relevante kriminologische Forschungsfelder in Bezug auf...

a) ...die Untersuchung der Täter („Verbrecher“)?

b) ...die Untersuchung von Deliktsphänomenen („Verbrechen“)?

Allgemein lässt sich für alle der genannten Themenbereiche feststellen, dass diese von einer erschöpfenden Behandlung deutlich entfernt sind und sich dort jeweils eine Vielzahl spannender Forschungsfelder finden lassen. Die folgenden Themen stellen insofern lediglich eine Auswahl an Fragen dar, die ich für in besonderem Maße relevant halte. $\mathrm{Zu}$ nächst sind - mit Feldern wie etwa Wirtschaftskriminalität oder völkerstrafrechtlichen Delikten - in den vergangenen 20 Jahren ganze Bereiche abweichenden Verhaltens wesentlich bedeutsamer bzw. sichtbarer geworden, die von der kriminologischen Forschung bislang nur in Teilen thematisiert wurden. Hier stellt sich jeweils eine Vielzahl von Forschungsfragen.

Im gleichen Zeitraum waren in der Bundesrepublik wie auch in den meisten anderen Staaten der westlichen Welt gravierende gesellschaftliche und wirtschaftliche Umbrüche zu verzeichnen. Während der damit verbundene Wandel gesellschaftlicher Bedingungen abweichenden Verhaltens und sozialer Kontrolle in der angloamerikanischen Diskussion ein verbreitetes Thema ist, findet eine entsprechende Debatte in der Bundesrepublik nur in Ansätzen statt.

Schließlich gilt nach wie vor, dass Entstehung und Ablauf von Kriminalisierungsprozessen - die mit ihren Aushandlungen und Zuschreibungen Handelnde zu Tätern und Geschehensabläufe zu Kriminalität machen - im Vergleich zu solchen Untersuchungen stark unterbelichtet sind, die diese Definitionen als Tatsache zugrunde legen und sich hiervon ausgehend der Untersuchung von Tat und Täter widmen.

c) ...die Untersuchung der staatlichen und gesellschaftlichen Reaktion auf Kriminalität („Verbrechenskontrolle")?

Hinsichtlich staatlicher Sozialkontrolle ist zunächst der Bereich des Strafrechts von Interesse. Neben dessen Ausweitung in den vergangenen drei Jahrzehnten, den diesbezüglichen Gesetzgebungsprozessen und Konjunkturen der Kriminalpolitik gilt dies insbesondere auch für die Rechtstatsachenforschung zum Strafverfahren und zur Tätigkeit der Strafverfolgungsinstanzen. Hier tun sich so verschiedene Felder auf wie lokale Justizkulturen, die Praxis der Strafzumessung bzw. allgemein richterlicher und staatsanwaltlicher Entscheidungen sowie detailliertere Analysen des Wandels der Strafverfolgungspraxis angesichts gesellschaftlicher Veränderungen, deren empirische Beforschung von hohem Interesse wäre.

Mindestens vergleichbare Lücken finden sich sodann bei neueren Formen staatlicher wie auch privater Sozialkontrolle, wie etwa technischer Überwachung und Kontrolle, situativer Kriminalprävention und Techniken der Selbstführung. Diese Entwicklungen

NK 25. Jg. $1 / 2013$ 
bedürfen zum einen weiterer grundlegender Betrachtung, etwa im Hinblick auf die ihnen innewohnende starke Präventionsorientierung und die Tendenz der Privatisierung. Zum anderen lassen sich die verschiedenen Formen aber auch für sich genommen untersuchen, wobei bislang vor allem die Videoüberwachung im Fokus stand.

Weiter vorangetrieben werden sollte schließlich auch das Themenfeld, das man zusammenfassend als gesellschaftliche Wahrnehmung abweichenden Verhaltens bezeichnen könnte. Hierunter lässt sich der Wandel gesellschaftlicher Vorstellungen über abweichendes Verhalten im Zeitablauf ebenso fassen, wie der Themenstrauß rund um die Aspekte subjektive Sicherheit und Kriminalitätsfurcht. Dies beinhaltet auch Verhältnis und Wechselwirkung dieser Phänomene mit anderen gesellschaftlichen Bereichen wie etwa Kriminalpolitik und Medien.

d) ...die Untersuchung der Rolle der Verbrechensopfer?

Wenngleich die Rolle des Opfers in der jüngeren Vergangenheit der Kriminologie eine vergleichsweise große Rolle gespielt hat, sind doch auch in diesem Bereich weitere vielversprechende Themen zu verzeichnen. So stellt sich etwa die Frage, wie die veränderte Rolle und Situation von Opfern und ihre gewandelte Stellung im Strafverfahren Kriminalisierungsprozesse beeinflussen. Weiter nachgegangen werden sollte auch der Frage, worin die Interessen von Opfern bei der Aufarbeitung von Viktimisierungsgeschehen liegen und welche Möglichkeiten es für die Befriedigung dieser Interessen auch außerhalb des Systems strafrechtlicher Sozialkontrolle gibt, das für diese Zwecke nur wenig geeignet scheint.

\section{Prof. Dr. Torsten Verrel, Universität Bonn:}

Wie bewerten Sie die aktuelle Lage und Bedeutung der Kriminologie in Deutschland?

Insgesamt führen wir wohl eher ein Randdasein. Rückgang in der universitären Abbildung durch entsprechende Lehrstühle, gleichgebliebene Wahrnehmungsprobleme in Kriminalpolitik und Medien; aber gewisse Einflussmöglichkeiten durch sachlich vorgetragene Argumente statt ständiger Blindheits-/Abendlanduntergangsrethorik. Zunehmendes Problem der Doppelgleisigkeit juristischer Kriminologen, mangelnde Medienkompetenz.

Wie würden Sie die Bedeutung der deutschen Kriminologie im internationalen Vergleich einordnen?

Da fehlen mir, ehrlich gesagt, die Einblicke ins Ausland. Ich vermute, dass wir aufgrund unserer Anbindung an die rechtswissenschaftliche Ausbildung einerseits nicht die Präsenz wie andere in großen Stil empirisch forschende sozialwissenschaftliche Kriminolo- 
gen haben. Andererseits scheinen mir die Einflussmöglichkeiten der ausländischen Kriminologen auf deren Kriminalpolitik keinesfalls größer zu sein. Also: klein, aber fein.

Wo sehen Sie Stärken und Schwächen der kriminologischen Ausbildung in Deutschland?

Zugang zu angehenden Juristen. Aufwertung in studentischer Wahrnehmung durch Schwerpunktbereiche, aber eben nicht mehr Bestandteil der staatlichen Prüfung und damit Gefahr der Bedeutungslosigkeit reiner Universitätsnoten im Vergleich zu (schlechteren) Pflichtfachnoten. Unzureichende Ausbildung in sozialwissenschaftlichen Methoden.

Wo sehen Sie auch in der Zukunft bedeutsame und gesellschaftlich sowie politisch relevante kriminologische Forschungsfelder in Bezug auf...

a) ...die Untersuchung der Täter („Verbrecher“)?

Theorieintegration, insb. neurowissenschaftlicher Befunde, noch stärkere Spezifizierung statt Universalansatz. Resilienzforschung

b) ...die Untersuchung von Deliktsphänomenen („Verbrechen“)?

Natürlich: digitale Revolution und Folgen, Cybercrime; neue Extremismusformen

c) ...die Untersuchung der staatlichen und gesellschaftlichen Reaktion auf Kriminalität („Verbrechenskontrolle“)?

Sicher ein ewiges Thema, da viel zu wenig an echter, sprich Ergebnis- und nicht bloßer Prozessevaluation; Beharrlichkeit in den Versuchen, die Öffentlichkeit mit nüchternen Informationen über Wirkungsforschung zu versorgen

d) ...die Untersuchung der Rolle der Verbrechensopfer?

Scheint mir kein Thema mit besonderem Zukunftspotential zu sein. 